\title{
Generalization of correlated electron-ion dynamics from nonequilibrium Green's functions
}

\author{
Yu Wang
}

October 24,2018

Atomistic Simulation Centre, School of Mathematics and Physics, Queen's University Belfast, Belfast BT7 1NN, United Kingdom

E-mail: yu.wang@qub.ac.uk

\begin{abstract}
We present a new formulation of the correlated electron-ion dynamics (CEID) by using equations of motion for nonequilibrium Green's functions, which generalizes CEID to a general nonequilibrium statistical ensemble that allows for a variable total number of electrons. We make a rigorous connection between CEID and diagrammatic perturbation theory, which furthermore allows the key approximations in CEID to be quantified in diagrammatic terms, and, in principle, improved. We compare analytically the limiting behavior of CEID and the self-consistent Born approximation (SCBA) for a general dynamical nonequilibrium state. This comparison shows that CEID and SCBA coincide in the weak electronphonon coupling limit, while they differ in the large ionic mass limit where we can readily quantify their difference. In particular, we illustrate the relation between CEID and SCBA by perturbation theory at the fourthorder in the coupling strength.
\end{abstract}




\section{Introduction}

One of the most fundamental problems in molecular electronics is to understand the inelastic scattering effects of atomic vibrations on transmitted electrons. These effects have been extensively studied both experimentally and theoretically in recent years [1]. The interplay between electronic and nuclear dynamics in atomic-scale devices influences not only the device characteristics, e.g. inelastic current-voltage spectroscopy [2], but also the device stability due to local heating within the junction [3].

Nonequilibrium Green's function (NEGF) theory [4, 5, 6] provides a systematic framework for describing the effects of the coupling between transmitted electrons and atomic vibrations $[7,8,9,10]$. For weak electron-phonon coupling, a well-known approximation for evaluating the dressed Green's function is the self-consistent Born approximation (SCBA) [8, 9, 10] which sums only noncrossing diagrams in the diagrammatic perturbation expansion of the Green's function. Because the percentage of noncrossing diagrams decreases quickly with increasing order in the coupling strength, SCBA breaks down at strong electron-phonon coupling [11].

Alternatively, correlated electron-ion dynamics (CEID) [2, 12, 13] has been developed for describing the effects of the electron-ion correlation and interaction on the inelastic dynamics of the electrons and nuclei. CEID, as an extension of molecular dynamics, reinstates the electron-ion correlation and the quantum nature of nuclei in order to take account of energy exchange between electrons and nuclei reasonably. So far CEID has been applied to a wide range of transport properties of atomic wires, including inelastic current-voltage spectroscopy [2], the calculation of local heating (and its signature on the current) in real time when combined with electronic open boundaries [14], and the non-conservative nature of current-induced forces [15]. Recently, a comparison between CEID and the NEGF method in SCBA has been made both numerically and analytically for steady state transport [16]. However, there are two restrictions on the original CEID discussed above. First, it is assumed that the electron-ion system is described in terms of an ensemble with a fixed total number of electrons. Second, the original CEID methodology lacks a systematic scheme to improve its accuracy.

In this paper, we develop a new formulation of CEID by using equations of motion for a set of nonequilibrium Green's functions which are closely linked to the dynamical variables in the CEID method. To illustrate this idea, we consider a model system of noninteracting electrons linearly coupled to a quantum oscillator. The motivation behind this effort is to lift the restrictions on the original CEID. We attempt to make a rigorous connection between CEID and diagrammatic perturbation theory, so as to quantify the key CEID approximations in diagrammatic terms, and, in principle, to be able to improve them. Moreover, in the framework of NEGF, the scope of CEID can be readily extended to a general nonequilibrium ensemble with a variable total number of electrons. We compare analytically CEID with SCBA for a general nonequilibrium state in

the time domain, thus extending the previous comparison [16] for a steady state 
in the energy domain.

The paper is organized as follows. In the next section, we present the new formulation of CEID for the model system by using equations of motion for a set of nonequilibrium Green's functions. In section 3, CEID is analytically compared with SCBA for the model system at the fourth-order in the coupling strength and in two specific limits: weak electron-phonon coupling limit and large ionic mass limit. To evaluate the Green's functions for the system of electrons coupled to a classical oscillator, a classical version of Wick's theorem is introduced in the appendix. Finally, conclusions are drawn in section 4

\section{Model and formulation}

We consider an infinite open system of noninteracting electrons linearly coupled to a single quantum oscillator. The electrons are described in terms of the second-quantized field operators $\Psi(r)$ and $\Psi^{+}(r)$. The Hamiltonian of the system then takes the form

$$
\begin{aligned}
H= & \int d r \Psi^{+}(r)\left(-\frac{\hbar^{2}}{2 m} \nabla^{2}+V(r)\right) \Psi(r)+\left[\frac{P^{2}}{2 M}+\frac{1}{2} K X^{2}\right] \\
& -X \int d r F(r) \Psi^{+}(r) \Psi(r)
\end{aligned}
$$

with $X=R-R_{0}$. The first two terms constitute the free-particle Hamiltonian $H_{0}$, and the last term describes the electron-phonon interaction $H^{i}$. Here $V(r)$ is the lattice potential and $F(r)$ is the electron-phonon coupling strength. $R_{0}$ and $K$ are the equilibrium position and the spring constant of the harmonic oscillator respectively.

In the absence of electron-phonon interaction $H^{i}$ (taken to exist at $t=$ $-\infty$ ), the unperturbed electron subsystem was settled in the Landauer steady state, which is characterized by two sets of one-electron states, i.e. LippmannSchwinger scattering states, $\left\{\left|\Phi_{i \alpha}\right\rangle\right\}(\alpha=1,2)$ with occupancies $f_{i \alpha}$ set by the battery terminals [17, 18]. In the $\left|\Phi_{i \alpha}\right\rangle$-representation, the statistical operator of the unperturbed system is thus taken to be

$$
\rho_{0}=\frac{1}{Z} \exp \left\{-\beta\left[\left(\frac{P^{2}}{2 M}+\frac{1}{2} K X^{2}\right)+\sum_{i} \sum_{\alpha=1}^{2}\left(\varepsilon_{i \alpha}-\mu_{\alpha}\right) c_{i \alpha}^{+} c_{i \alpha}\right]\right\}
$$

where $\beta=1 / k_{B} T$ is the inverse temperature, electrons occupying the two sets of Lippmann-Schwinger scattering states are characterized by the chemical potentials $\mu_{\alpha}(\alpha=1,2)$ respectively, and $Z$ is a normalization factor ensuring that $\operatorname{Tr}\left(\rho_{0}\right)=1$. Here, $\left\{c_{i \alpha}^{+}\right\}$and $\left\{c_{i \alpha}\right\}$ are the creation and annihilation operators for the complete and orthonormal set of the Lippmann-Schwinger scattering states $\left\{\left|\Phi_{i \alpha}\right\rangle\right\}$. The fermion field operator $\Psi(r)\left(\Psi^{+}(r)\right)$ can thus be expressed as a linear combination of $\left\{c_{i \alpha}\right\}\left(\left\{c_{i \alpha}^{+}\right\}\right)$.

We now define the contour-ordered Green's function 


$$
G\left(r t, r^{\prime} t^{\prime}\right)=(i \hbar)^{-1}\left\langle T_{C} \psi_{H}(r t) \psi_{H}^{+}\left(r^{\prime} t^{\prime}\right)\right\rangle
$$

where the angular bracket $\langle\cdots\rangle=\operatorname{tr}\left(\rho_{0} \cdots\right)$. By virtue of the grand-canonical structure of $\rho_{0}$, we have thus allowed for an ensemble with a variable total number of electrons. The contour $C$ runs from $t=-\infty$ to $t=\infty$ along the upper branch and then returns to $t=-\infty$ along the lower branch. Here $\psi_{H}(r t)$ and $\psi_{H}^{+}\left(r^{\prime} t^{\prime}\right)$ are the fermion field operators in the Heisenberg picture.

Parallel to the original procedure of CEID [16], our main aim is to derive the kinetic equation for the one-electron density matrix. Then the key quantity of interest is the lesser Green's function $G^{<}\left(r t, r^{\prime} t^{\prime}\right)=-(i \hbar)^{-1}\left\langle\psi_{H}^{+}\left(r^{\prime} t^{\prime}\right) \psi_{H}(r t)\right\rangle$ since its equal-time value gives the one-electron density matrix:

$$
\rho_{e}\left(r, t \mid r^{\prime}, t\right)=-i \hbar G^{<}\left(r t, r^{\prime} t\right)=\left\langle\psi_{H}^{+}\left(r^{\prime} t\right) \psi_{H}(r t)\right\rangle
$$

We first derive the equation of motion for the contour-ordered Green's function $G\left(r t, r^{\prime} t^{\prime}\right)$. Differentiating $G\left(r t, r^{\prime} t^{\prime}\right)$ with respect to time arguments and then using equations of motion for the Heisenberg operators, one obtains

$$
\begin{gathered}
\left(i \hbar \partial_{t}-h_{e}(r)\right) G\left(r t, r^{\prime} t^{\prime}\right)=\delta\left(r-r^{\prime}\right) \delta_{C}\left(t-t^{\prime}\right)-F(r) \Gamma_{\mu}\left(r t, r^{\prime} t^{\prime}\right) \\
\left(-i \hbar \partial_{t^{\prime}}-h_{e}\left(r^{\prime}\right)\right) G\left(r t, r^{\prime} t^{\prime}\right)=\delta\left(r-r^{\prime}\right) \delta_{C}\left(t-t^{\prime}\right)-\Gamma_{\mu}^{\prime}\left(r t, r^{\prime} t^{\prime}\right) F\left(r^{\prime}\right)
\end{gathered}
$$

where $h_{e}(r)=-\frac{\hbar^{2}}{2 m} \nabla^{2}+V(r)$ and $\delta_{C}\left(t-t^{\prime}\right)$ is the contour delta function [6]. Two new nonequilibrium Green's functions are introduced here

$$
\begin{aligned}
& \Gamma_{\mu}\left(r t, r^{\prime} t^{\prime}\right)=(i \hbar)^{-1}\left\langle T_{C} X_{H}(t) \psi_{H}(r t) \psi_{H}^{+}\left(r^{\prime} t^{\prime}\right)\right\rangle \\
& \Gamma_{\mu}^{\prime}\left(r t, r^{\prime} t^{\prime}\right)=(i \hbar)^{-1}\left\langle T_{C} \psi_{H}(r t) \psi_{H}^{+}\left(r^{\prime} t^{\prime}\right) X_{H}\left(t^{\prime}\right)\right\rangle
\end{aligned}
$$

Combining equations (44) and (15) gives

$$
\dot{\rho}_{e}=\frac{1}{i \hbar}\left[h_{e}, \rho_{e}\right]-\frac{1}{i \hbar}[F, \mu]
$$

where we used $\dot{\rho}_{e}\left(r, t \mid r^{\prime}, t\right)=-i \hbar \lim _{t \rightarrow t^{\prime}}\left[\left(\partial_{t}+\partial_{t^{\prime}}\right) G\left(r t, r^{\prime} t^{\prime}\right)\right]^{<}$, and the first moment $\mu\left(r, t \mid r^{\prime}, t\right)$ is defined as:

$$
\begin{aligned}
\mu\left(r, t \mid r^{\prime}, t\right) & \equiv-i \hbar \Gamma_{\mu}^{<}\left(r t, r^{\prime} t\right)=-i \hbar \Gamma_{\mu}^{<}\left(r t, r^{\prime} t\right) \\
& =\left\langle\psi_{H}^{+}\left(r^{\prime} t\right) \psi_{H}(r t) X_{H}(t)\right\rangle
\end{aligned}
$$

Note that the kinetic equation (8) always ensures the conservation of electron number, since the relation $\left\langle\dot{N}_{e}\right\rangle=\operatorname{tr} \dot{\rho}_{e}=0$ holds due to the cyclic invariance of the trace. 
We proceed to find the equations of motion for $\Gamma_{\mu}\left(r t, r^{\prime} t^{\prime}\right)$ :

$$
\begin{gathered}
\left(i \hbar \partial_{t}-h_{e}(r)\right) \Gamma_{\mu}\left(r t, r^{\prime} t^{\prime}\right)=\delta\left(r-r^{\prime}\right) \delta_{C}\left(t-t^{\prime}\right)\left\langle X_{H}(t)\right\rangle-F(r) \Gamma_{\mu_{2}}\left(r t, r^{\prime} t^{\prime}\right)+\frac{i \hbar}{M} \Gamma_{\lambda}\left(r t, r^{\prime} t^{\prime}\right) \\
\left(-i \hbar \partial_{t^{\prime}}-h_{e}\left(r^{\prime}\right)\right) \Gamma_{\mu}\left(r t, r^{\prime} t^{\prime}\right)=\delta\left(r-r^{\prime}\right) \delta_{C}\left(t-t^{\prime}\right)\left\langle X_{H}(t)\right\rangle-\Gamma_{\mu_{2}}^{\prime}\left(r t, r^{\prime} t^{\prime}\right) F\left(r^{\prime}\right)
\end{gathered}
$$

where three new nonequilibrium Green's functions are defined as:

$$
\begin{gathered}
\Gamma_{\lambda}\left(r t, r^{\prime} t^{\prime}\right)=(i \hbar)^{-1}\left\langle T_{C} P_{H}(t) \psi_{H}(r t) \psi_{H}^{+}\left(r^{\prime} t^{\prime}\right)\right\rangle \\
\Gamma_{\mu_{2}}\left(r t, r^{\prime} t^{\prime}\right)=(i \hbar)^{-1}\left\langle T_{C} X_{H}^{2}(t) \psi_{H}(r t) \psi_{H}^{+}\left(r^{\prime} t^{\prime}\right)\right\rangle \\
\Gamma_{\mu_{2}}^{\prime}\left(r t, r^{\prime} t^{\prime}\right)=(i \hbar)^{-1}\left\langle T_{C} X_{H}(t) \psi_{H}(r t) \psi_{H}^{+}\left(r^{\prime} t^{\prime}\right) X_{H}\left(t^{\prime}\right)\right\rangle
\end{gathered}
$$

In order to obtain a closed set of equations of motion, we decouple the higherorder Green's functions $\Gamma_{\mu_{2}}\left(r t, r^{\prime} t^{\prime}\right)$ and $\Gamma_{\mu_{2}}^{\prime}\left(r t, r^{\prime} t^{\prime}\right)$ as follows:

$$
\begin{gathered}
\Gamma_{\mu_{2}}\left(r t, r^{\prime} t^{\prime}\right) \approx C_{R R}(t) G\left(r t, r^{\prime} t^{\prime}\right) \\
\Gamma_{\mu_{2}}^{\prime}\left(r t, r^{\prime} t^{\prime}\right) \approx i \hbar D\left(t, t^{\prime}\right) G\left(r t, r^{\prime} t^{\prime}\right)
\end{gathered}
$$

where $D\left(t, t^{\prime}\right)=(i \hbar)^{-1}\left\langle T_{C} X_{H}(t) X_{H}\left(t^{\prime}\right)\right\rangle$ is the dressed phonon Green's function and $C_{R R}(t)=i \hbar D^{<}(t, t)$. Using these decoupling approximations and the relation $\dot{\mu}\left(r, t \mid r^{\prime}, t\right)=-i \hbar \lim _{t \rightarrow t^{\prime}}\left[\left(\partial_{t}+\partial_{t^{\prime}}\right) \Gamma_{\mu}\left(r t, r^{\prime} t^{\prime}\right)\right]^{<}$, we combine equations (10) and (11) to yield

$$
\dot{\mu}=\frac{1}{i \hbar}\left[h_{e}, \mu\right]-\frac{1}{i \hbar} C_{R R}\left[F, \rho_{e}\right]+\frac{\lambda}{M}
$$

where we have applied the Langreth theorem [5, 6] to calculate $\Gamma_{\mu_{2}}^{<<}\left(r t, r^{\prime} t^{\prime}\right)=$ $i \hbar D^{<}\left(t, t^{\prime}\right) G^{<}\left(r t, r^{\prime} t^{\prime}\right)$, and the first moment $\lambda\left(r, t \mid r^{\prime}, t\right)$ is defined as

$$
\begin{aligned}
\lambda\left(r, t \mid r^{\prime}, t\right) & \equiv-i \hbar \Gamma_{\lambda}^{<}\left(r t, r^{\prime} t\right) \\
& =\left\langle\psi_{H}^{+}\left(r^{\prime} t\right) \psi_{H}(r t) P_{H}(t)\right\rangle
\end{aligned}
$$

We continue to derive the equation of motion for $\Gamma_{\lambda}\left(r t, r^{\prime} t^{\prime}\right)$ :

$$
\begin{aligned}
& \left(i \hbar \partial_{t}-h_{e}(r)\right) \Gamma_{\lambda}\left(r t, r^{\prime} t^{\prime}\right)=\delta\left(r-r^{\prime}\right) \delta_{C}\left(t-t^{\prime}\right)\left\langle P_{H}(t)\right\rangle-i \hbar K \Gamma_{\mu}\left(r t, r^{\prime} t^{\prime}\right) \\
& +\Lambda\left(r t, r^{\prime} t^{\prime}\right)-F(r) \Gamma_{\lambda \mu}\left(r t, r^{\prime} t^{\prime}\right) \\
& \left(-i \hbar \partial_{t^{\prime}}-h_{e}\left(r^{\prime}\right)\right) \Gamma_{\lambda}\left(r t, r^{\prime} t^{\prime}\right)=\delta\left(r-r^{\prime}\right) \delta_{C}\left(t-t^{\prime}\right)\left\langle P_{H}(t)\right\rangle-\Gamma_{\lambda \mu}^{\prime}\left(r t, r^{\prime} t^{\prime}\right) F\left(r^{\prime}\right)
\end{aligned}
$$


where three new nonequilibrium Green's functions are defined as:

$$
\begin{gathered}
\Lambda\left(r t, r^{\prime} t^{\prime}\right)=\int d r_{0} F\left(r_{0}\right)\left\langle T_{C} \psi_{H}^{+}\left(r_{0} t\right) \psi_{H}\left(r_{0} t\right) \psi_{H}(r t) \psi_{H}^{+}\left(r^{\prime} t^{\prime}\right)\right\rangle \\
\Gamma_{\lambda \mu}\left(r t, r^{\prime} t^{\prime}\right)=(i \hbar)^{-1}\left\langle T_{C} P_{H}(t) X_{H}(t) \psi_{H}(r t) \psi_{H}^{+}\left(r^{\prime} t^{\prime}\right)\right\rangle \\
\Gamma_{\lambda \mu}^{\prime}\left(r t, r^{\prime} t^{\prime}\right)=(i \hbar)^{-1}\left\langle T_{C} P_{H}(t) \psi_{H}(r t) \psi_{H}^{+}\left(r^{\prime} t^{\prime}\right) X_{H}\left(t^{\prime}\right)\right\rangle
\end{gathered}
$$

To decouple the above higher-order Green's functions, we make the following approximations:

$$
\begin{gathered}
\Lambda\left(r t, r^{\prime} t^{\prime}\right) \approx \int d r_{0} F\left(r_{0}\right)\left\langle\psi_{H}^{+}\left(r_{0} t\right) \psi_{H}\left(r_{0} t\right)\right\rangle\left\langle T_{C} \psi_{H}(r t) \psi_{H}^{+}\left(r^{\prime} t^{\prime}\right)\right\rangle \\
-\int d r_{0}\left\langle\psi_{H}^{+}\left(r_{0} t\right) \psi_{H}(r t)\right\rangle F\left(r_{0}\right)\left\langle T_{C} \psi_{H}\left(r_{0} t\right) \psi_{H}^{+}\left(r^{\prime} t^{\prime}\right)\right\rangle \\
=i \hbar \int d r_{0} F\left(r_{0}\right) \rho_{e}\left(r_{0}, t\right) G\left(r t, r^{\prime} t^{\prime}\right)-i \hbar \int d r_{0} \rho_{e}\left(r, t \mid r_{0}, t\right) F\left(r_{0}\right) G\left(r_{0} t, r^{\prime} t^{\prime}\right) \\
\begin{array}{c}
\Gamma_{\lambda \mu}\left(r t, r^{\prime} t^{\prime}\right) \approx(i \hbar)^{-1}\left\langle P_{H}(t) X_{H}(t)\right\rangle\left\langle T_{C} \psi_{H}(r t) \psi_{H}^{+}\left(r^{\prime} t^{\prime}\right)\right\rangle \\
=C_{P R}(t) G\left(r t, r^{\prime} t^{\prime}\right)-\frac{i \hbar}{2} G\left(r t, r^{\prime} t^{\prime}\right) \\
\Gamma_{\lambda \mu}^{\prime}\left(r t, r^{\prime} t^{\prime}\right) \approx\left\langle T_{C} P_{H}(t) X_{H}\left(t^{\prime}\right)\right\rangle G\left(r t, r^{\prime} t^{\prime}\right)
\end{array}
\end{gathered}
$$

where $C_{P R}(t)=\frac{1}{2}\left\langle P_{H}(t) X_{H}(t)+X_{H}(t) P_{H}(t)\right\rangle$. Using the above decoupling approximations and the relation $\dot{\lambda}\left(r, t \mid r^{\prime}, t\right)=-i \hbar \lim _{t \rightarrow t^{\prime}}\left[\left(\partial_{t}+\partial_{t^{\prime}}\right) \Gamma_{\lambda}\left(r t, r^{\prime} t^{\prime}\right)\right]^{<}$, we combine equations (19) and (20) to arrive at

$$
\dot{\lambda}=\frac{1}{i \hbar}\left[h_{e}, \lambda\right]+\operatorname{tr}\left(\rho_{e} F\right) \rho_{e}+\frac{1}{2}\left\{F, \rho_{e}\right\}-\rho_{e} F \rho_{e}-\frac{1}{i \hbar} C_{P R}\left[F, \rho_{e}\right]-K \mu
$$

where the Langreth theorem is applied to calculate $\Gamma_{\lambda \mu}^{\prime<}\left(r t, r^{\prime} t^{\prime}\right)=\left\langle X_{H}\left(t^{\prime}\right) P_{H}(t)\right\rangle G^{<}\left(r t, r^{\prime} t^{\prime}\right)$.

Kinetic equations (8), (17) and (27) are identical to those derived in Refs. [2, 16], except that an extra term $\operatorname{tr}\left(\rho_{e} F\right) \rho_{e}$ appears and a $\mu F \mu$ term disappears in equation (27) compared with the corresponding equation in the original CEID. The reason for the presence of the extra $\operatorname{tr}\left(\rho_{e} F\right) \rho_{e}$ term is that, in the original CEID [2, 16], the expansion was with respect to $\Delta R=R-\bar{R}$, whereas we, for convenience, here use $X=R-R_{0}$ instead. In the original CEID, the $\mu F \mu$ term results from higher-order corrections to the Hartree-Fock decoupling for the twoelectron density matrix in equation (24), which, however is not considered in the present formulation. Apart from the two differences, the present formulation of CEID is parallel to the original CEID. 
In order to have a closed set of equations, one can derive the perturbation expansion for $C_{R R}(t)$ and $C_{P R}(t)$ by using the expression [4]

$$
\left\langle A_{H}(t) B_{H}(t)\right\rangle=\left\langle T_{C}\left[e^{-\frac{i}{\hbar} \int_{C} H_{H_{0}}^{i}(\tau) d \tau} A_{H_{0}}(t) B_{H_{0}}(t)\right]\right\rangle
$$

It is obvious that Wick's theorem can be applied directly to the $T_{C}$-products of $\psi_{H_{0}}$ 's and $\psi_{H_{0}}^{+}$'s. For simplicity, we shall only retain the zero-order term in the expansion. Thus we set $C_{R R}(t)=i \hbar D_{0}^{<}(t, t)=C$ (C is a constant) and $C_{P R}(t)=\frac{1}{2}\left\langle P_{H_{0}}(t) X_{H_{0}}(t)+X_{H_{0}}(t) P_{H_{0}}(t)\right\rangle=0$. We shall hereafter consider the bare phonon Green's function $D_{0}\left(t, t^{\prime}\right)$ only, instead of the dressed one $D\left(t, t^{\prime}\right)$.

The decoupling approximations (15), (16), (24), (25) and (26) are the defining approximations in the original CEID method [2]. Their key effect is to yield single-time equations of motion. These approximations can be well understood in the framework of diagrammatic perturbation theory as follows. Each of them represents a subset of diagrams in the diagrammatic perturbation expansion of the corresponding Green's function. As shown in figure 1 where we use $\Gamma_{\mu_{2}}^{\prime}\left(r t, r^{\prime} t^{\prime}\right)$ as an example, the decoupling approximation (16) includes the first diagram in figure 1(a) and consequently coincides exactly with the exact perturbation expansion at the lowest-order, while it includes only the second diagram in figure 1 (a) at the second-order.

In the NEGF-based formulation, CEID can be systematically extended in two possible ways. Following the standard equation-of-motion technique, one may extend the hierarchy of coupled equations of motion for Green's functions and then truncate the hierarchy somewhere by some sort of decoupling procedure in which a higher-order Green's functions is expressed approximately as a product of lower-order Green's functions. Another possibility is to improve the decoupling approximations for Green's functions $\Gamma_{\mu_{2}}, \Gamma_{\mu_{2}}^{\prime}, \Lambda, \Gamma_{\lambda \mu}$ and $\Gamma_{\lambda \mu}^{\prime}$ by adding correction terms. For instance, the last diagram in figure 1(a) (a second-order noncrossing diagram in the exact perturbation expansion for $\Gamma_{\mu_{2}}^{\prime}$ ) which is absent from figure 1 (b) serves as a second-order correction term to the decoupling approximation (16) illustrated in figure 1(b). We shall focus on the way of making corrections to CEID decoupling approximations, since it does not result in a higher hierarchy of coupled equations (see section 3.2).

We have thus rederived the CEID equations of motion from nonequilibrium Green's functions and generalized them to an ensemble which allows for a variable total number of electrons. Moreover, the present formulation allows the key approximations in CEID to be quantified in diagrammatic terms and provides an in-principle way to improve them.

\section{Comparison with the self-consistent Born ap- proximation}

\subsection{Weak electron-phonon coupling limit}

The CEID equations of motion to lowest-order in $F$ read: 


$$
\begin{gathered}
\dot{\rho}_{e}^{(2)}=\frac{1}{i \hbar}\left[h_{e}, \rho_{e}^{(2)}\right]-\frac{1}{i \hbar}\left[F, \mu^{(1)}\right] \\
\dot{\mu}^{(1)}=\frac{1}{i \hbar}\left[h_{e}, \mu^{(1)}\right]-\frac{1}{i \hbar} C\left[F, \rho_{e}^{(0)}\right]+\frac{\lambda^{(1)}}{M} \\
\dot{\lambda}^{(1)}=\frac{1}{i \hbar}\left[h_{e}, \lambda^{(1)}\right]+\operatorname{tr}\left(\rho_{e}^{(0)} F\right) \rho_{e}^{(0)}+\frac{1}{2}\left\{F, \rho_{e}^{(0)}\right\}-\rho_{e}^{(0)} F \rho_{e}^{(0)}-K \mu^{(1)}
\end{gathered}
$$

where the superscript denotes the order in the coupling strength $F$. Note that the decoupling approximations (15), (16), (24), (25) and (26) are exact to lowestorder in $F$. Hence the above kinetic equations yield the exact $\rho_{e}^{(2)}$. According to equation (3), the density matrix $\rho_{e}^{(2)}$ must correspond to the sum of all the second-order terms (the Hartree and Fock diagrams) in the perturbation expansion of Green's function $G\left(1,1^{\prime}\right)$, which reads

$$
\begin{aligned}
& G^{(2)}\left(1,1^{\prime}\right)=\operatorname{tr}\left(\rho_{e}^{(0)} F\right) \int d 2 d t_{3} D_{0}\left(t_{2}, t_{3}\right) G_{0}(1,2) F\left(r_{2}\right) G_{0}\left(2,1^{\prime}\right) \\
& \quad+i \hbar \int d 2 d 3 G_{0}(1,2) F\left(r_{2}\right) D_{0}\left(t_{2}, t_{3}\right) G_{0}(2,3) F\left(r_{3}\right) G_{0}\left(3,1^{\prime}\right)
\end{aligned}
$$

Here we use a common short notation $(k) \equiv\left(r_{k} t_{k}\right)$. Starting from this equation, one can also easily derive equations (28), (29) and (30) with the equation-ofmotion technique, and furthermore identify that the second term in the righthand side of equation (30) comes from the contribution of the Hartree term.

Recall that in SCBA the sum of the second-order terms $G_{S C B A}^{(2)}$, i.e. the first Born approximation, involves both the Hartree and Fock diagrams and coincides exactly with equation (31). Hence, in the weak electron-phonon coupling limit CEID agrees exactly with SCBA for an arbitrary nonequilibrium state of the electron-ion system, which extends the range of validity of the conclusion in the previous comparison [16] for a steady state in the energy domain.

\subsection{The fourth-order in the coupling strength}

We shall go beyond the weak electron-phonon coupling limit and compare CEID and SCBA at the fourth-order in $F$. For simplicity, Hartree-like diagrams (with a closed fermion loop) will be excluded from our analysis. Let us consider the fourth-order SCBA Green's function $G^{(4)}\left(1,1^{\prime}\right)=A_{1}\left(1,1^{\prime}\right)+A_{2}\left(1,1^{\prime}\right)$ which is represented by two diagrams:

$$
\begin{aligned}
& A_{1}\left(1,1^{\prime}\right)=i \hbar \int d 2 d 3 G_{0}(1,2) F\left(r_{2}\right) D_{0}\left(t_{2}, t_{3}\right) G_{0}(2,3) F\left(r_{3}\right) G^{(2)}\left(3,1^{\prime}\right) \\
& A_{2}\left(1,1^{\prime}\right)=i \hbar \int d 2 d 3 G_{0}(1,2) F\left(r_{2}\right) D_{0}\left(t_{2}, t_{3}\right) G^{(2)}(2,3) F\left(r_{3}\right) G_{0}\left(3,1^{\prime}\right)
\end{aligned}
$$


These diagrams are shown in figure $2(\mathrm{a})$ and (b) respectively. Here $G^{(2)}\left(1,1^{\prime}\right)=$ $i \hbar \int d 2 d 3 G_{0}(1,2) F\left(r_{2}\right) D_{0}\left(t_{2}, t_{3}\right) G_{0}(2,3) F\left(r_{3}\right) G_{0}\left(3,1^{\prime}\right)$ since the Hartree diagram is ignored. Kinetic equations for $G^{(4)}\left(1,1^{\prime}\right)$ with respect to $t_{1}$ and $t_{1}^{\prime}$ yield $\dot{\rho}_{e}^{(4)}=(i \hbar)^{-1}\left[h_{e}, \rho_{e}^{(4)}\right]-(i \hbar)^{-1}\left[F, \mu^{(3)}\right]$ where the corresponding $\Gamma_{\mu}^{(3)}\left(1,1^{\prime}\right)=$ $B_{1}\left(1,1^{\prime}\right)+B_{2}\left(1,1^{\prime}\right)$ contains two terms:

$$
\begin{aligned}
& B_{1}\left(1,1^{\prime}\right)=-i \hbar \int d 2 D_{0}\left(t_{1}, t_{2}\right) G_{0}(1,2) F\left(r_{2}\right) G^{(2)}\left(2,1^{\prime}\right) \\
& B_{2}\left(1,1^{\prime}\right)=-i \hbar \int d 2 D_{0}\left(t_{1}, t_{2}\right) G^{(2)}(1,2) F\left(r_{2}\right) G_{0}\left(2,1^{\prime}\right)
\end{aligned}
$$

Note that $B_{1}\left(1,1^{\prime}\right)$ and $B_{2}\left(1,1^{\prime}\right)$ result from the temporal derivatives of $A_{1}\left(1,1^{\prime}\right)$ and $A_{2}\left(1,1^{\prime}\right)$ respectively. The kinetic equations for $\Gamma_{\mu}^{(3)}\left(1,1^{\prime}\right)$ read

$$
\begin{aligned}
& i \hbar \partial_{t_{1}} \Gamma_{\mu}^{(3)}\left(1,1^{\prime}\right)=h_{e}\left(r_{1}\right) \Gamma_{\mu}^{(3)}\left(1,1^{\prime}\right)-F\left(r_{1}\right)\left[C G^{(2)}\left(1,1^{\prime}\right)+S_{1}\left(1,1^{\prime}\right)\right]+i \hbar \frac{\Gamma_{\lambda}^{(3)}\left(1,1^{\prime}\right)}{M_{(32)}} \\
& -i \hbar \partial_{t_{1}^{\prime}} \Gamma_{\mu}^{(3)}\left(1,1^{\prime}\right)=\Gamma_{\mu}^{(3)}\left(1,1^{\prime}\right) h_{e}\left(r_{1}^{\prime}\right)-\left[i \hbar D_{0}\left(t_{1}, t_{1}^{\prime}\right) G^{(2)}\left(1,1^{\prime}\right)+S_{2}\left(1,1^{\prime}\right)\right] F\left(r_{1}^{\prime}\right)
\end{aligned}
$$

where $S_{1}\left(1,1^{\prime}\right)=(i \hbar)^{2} \int d 2 d 3 D_{0}\left(t_{1}, t_{3}\right) D_{0}\left(t_{1}, t_{2}\right) G_{0}(1,2) F\left(r_{2}\right) G_{0}(2,3) F\left(r_{3}\right) G_{0}\left(3,1^{\prime}\right)$, $S_{2}\left(1,1^{\prime}\right)=(i \hbar)^{2} \int d 2 d 3 D_{0}\left(t_{1}, t_{2}\right) G_{0}(1,2) F\left(r_{2}\right) G_{0}(2,3) F\left(r_{3}\right) D_{0}\left(t_{3}, t_{1}^{\prime}\right) G_{0}\left(3,1^{\prime}\right)$, and $\Gamma_{\lambda}^{(3)}\left(1,1^{\prime}\right)=C_{1}\left(1,1^{\prime}\right)+C_{2}\left(1,1^{\prime}\right)$ where

$$
\begin{aligned}
& C_{1}\left(1,1^{\prime}\right)=-i \hbar \int d 2 G_{0}(1,2) d_{0}\left(t_{1}, t_{2}\right) F\left(r_{2}\right) G^{(2)}\left(2,1^{\prime}\right) \\
& C_{2}\left(1,1^{\prime}\right)=-i \hbar \int d 2 d_{0}\left(t_{1}, t_{2}\right) G^{(2)}(1,2) F\left(r_{2}\right) G_{0}\left(2,1^{\prime}\right)
\end{aligned}
$$

with $d_{0}\left(t, t^{\prime}\right)=(i \hbar)^{-1}\left\langle T_{C} P_{H_{0}}(t) X_{H_{0}}\left(t^{\prime}\right)\right\rangle$. The two terms in the square bracket in equation (32) (equation (33)) correspond to all the second-order noncrossing diagrams in $\Gamma_{\mu_{2}}\left(1,1^{\prime}\right)\left(\Gamma_{\mu_{2}}^{\prime}\left(1,1^{\prime}\right)\right)$ (see equations (13) and (14)), while $C G^{(2)}\left(1,1^{\prime}\right)$ $\left(i \hbar D_{0}\left(t_{1}, t_{1}^{\prime}\right) G^{(2)}\left(1,1^{\prime}\right)\right)$ corresponds to the single second-order diagram in the decoupling approximation for $\Gamma_{\mu_{2}}\left(1,1^{\prime}\right)\left(\Gamma_{\mu_{2}}^{\prime}\left(1,1^{\prime}\right)\right)$ (see equations (15) and (16)) where $S_{1}\left(1,1^{\prime}\right)\left(S_{2}\left(1,1^{\prime}\right)\right)$ is not present. This was illustrated in figure 1 , where $i \hbar D_{0}\left(t_{1}, t_{1}^{\prime}\right) G^{(2)}\left(1,1^{\prime}\right)$ and $S_{2}\left(1,1^{\prime}\right)$ in equation (33) are associated with the second and third diagrams in figure 1(a) respectively. Equations (32) and (33) lead to

$$
\dot{\mu}^{(3)}=\frac{1}{i \hbar}\left[h_{e}, \mu^{(3)}\right]-\frac{1}{i \hbar} C\left[F, \rho_{e}^{(2)}\right]+\frac{\lambda^{(3)}}{M}+\Pi_{\mu}
$$

where $\Pi_{\mu}\left(r, t \mid r^{\prime}, t\right)=F(r) S_{1}^{<}\left(r t, r^{\prime} t\right)-S_{2}^{<}\left(r t, r^{\prime} t\right) F\left(r^{\prime}\right)$. It is seen that $\Pi_{\mu}$, contributed by the diagrams $S_{1}\left(1,1^{\prime}\right)$ and $S_{2}\left(1,1^{\prime}\right)$, serves as a correction to the 
CEID equation of motion for $\mu^{(3)}$ (cf. equation (17) at the third-order), so as to make CEID equivalent to SCBA at the fourth-order in the coupling strength. However this correction no longer involves just single-time quantities.

One can proceed to analyze the kinetic equations for $\Gamma_{\lambda}^{(3)}\left(1,1^{\prime}\right)$ in a similar manner:

$$
\begin{aligned}
i \hbar \partial_{t_{1}} \Gamma_{\lambda}^{(3)}\left(1,1^{\prime}\right)= & h_{e}\left(r_{1}\right) \Gamma_{\lambda}^{(3)}\left(1,1^{\prime}\right)-F\left(r_{1}\right)\left[\left\langle P_{H_{0}}\left(t_{1}\right) X_{H_{0}}\left(t_{1}\right)\right\rangle G^{(2)}\left(1,1^{\prime}\right)+S_{3}\left(1,1^{\prime}\right)\right] \\
& -i \hbar \int d r_{0} \rho_{e}^{(0)}\left(r_{1}, t_{1} \mid r_{0}, t_{1}\right) F\left(r_{0}\right) G^{(2)}\left(r_{0} t_{1}, r_{1}^{\prime} t_{1}^{\prime}\right) \\
& -i \hbar \int d r_{0} \rho_{e}^{(2)}\left(r_{1}, t_{1} \mid r_{0}, t_{1}\right) F\left(r_{0}\right) G_{0}\left(r_{0} t_{1}, r_{1}^{\prime} t_{1}^{\prime}\right)-i \hbar K \Gamma_{\mu}^{(3)}\left(1,1^{\prime}\right)(34) \\
-i \hbar \partial_{t_{1}^{\prime}} \Gamma_{\lambda}^{(3)}\left(1,1^{\prime}\right)= & \Gamma_{\lambda}^{(3)}\left(1,1^{\prime}\right) h_{e}\left(r_{1}^{\prime}\right)-\left[i \hbar d_{0}\left(t_{1}, t_{1}^{\prime}\right) G^{(2)}\left(1,1^{\prime}\right)+S_{4}\left(1,1^{\prime}\right)\right] F\left(r_{1}^{\prime}\right)
\end{aligned}
$$

where $S_{3}\left(1,1^{\prime}\right)=(i \hbar)^{2} \int d 2 d 3 d_{0}\left(t_{1}, t_{3}\right) D_{0}\left(t_{1}, t_{2}\right) G_{0}(1,2) F\left(r_{2}\right) G_{0}(2,3) F\left(r_{3}\right) G_{0}\left(3,1^{\prime}\right)$ and $S_{4}\left(1,1^{\prime}\right)=(i \hbar)^{2} \int d 2 d 3 d_{0}\left(t_{1}, t_{2}\right) G_{0}(1,2) F\left(r_{2}\right) G_{0}(2,3) F\left(r_{3}\right) D_{0}\left(t_{3}, t_{1}^{\prime}\right) G_{0}\left(3,1^{\prime}\right)$.

The two terms in the square bracket in equation (34) (equation (35)) correspond to all the second-order noncrossing diagrams in $\Gamma_{\lambda \mu}\left(1,1^{\prime}\right)\left(\Gamma_{\lambda \mu}^{\prime}\left(1,1^{\prime}\right)\right)$ (see equations (22) and (23)), while $\left\langle P_{H_{0}}\left(t_{1}\right) X_{H_{0}}\left(t_{1}\right)\right\rangle G^{(2)}\left(1,1^{\prime}\right)\left(i \hbar d_{0}\left(t_{1}, t_{1}^{\prime}\right) G^{(2)}\left(1,1^{\prime}\right)\right)$ is the single second-order diagram in the decoupling approximation for $\Gamma_{\lambda \mu}\left(1,1^{\prime}\right)$ $\left(\Gamma_{\lambda \mu}^{\prime}\left(1,1^{\prime}\right)\right)$ (see equations (25) and (26) ) where $S_{3}\left(1,1^{\prime}\right)\left(S_{4}\left(1,1^{\prime}\right)\right)$ is not present.

From equations (34) and (35), one obtains

$$
\dot{\lambda}^{(3)}=\frac{1}{i \hbar}\left[h_{e}, \lambda^{(3)}\right]+\frac{1}{2}\left\{F, \rho_{e}^{(2)}\right\}-\left(\rho_{e}^{(0)} F \rho_{e}^{(2)}+\rho_{e}^{(2)} F \rho_{e}^{(0)}\right)-K \mu^{(3)}+\Pi_{\lambda}
$$

where $\Pi_{\lambda}\left(r, t \mid r^{\prime}, t\right)=F(r) S_{3}^{<}\left(r t, r^{\prime} t\right)-S_{4}^{<}\left(r t, r^{\prime} t\right) F\left(r^{\prime}\right)$ which serves as a correction to the CEID equation of motion for $\lambda^{(3)}$ (cf. equation (27) at the thirdorder). Note that a term similar to the second term in the right-hand side of equation (30) does not appear because of the exclusion of Hartree-like diagrams.

We have thus identified explicitly the corrections to the CEID equations of motion for $\mu^{(3)}$ and $\lambda^{(3)}$ added by SCBA. These correction terms are contributed by the diagrams which are absent from the decoupling approximation but are present in the complete collection of second-order noncrossing diagrams for higher-order Green's functions $\Gamma_{\mu_{2}}\left(1,1^{\prime}\right), \Gamma_{\mu_{2}}^{\prime}\left(1,1^{\prime}\right), \Gamma_{\lambda \mu}\left(1,1^{\prime}\right)$ and $\Gamma_{\lambda \mu}^{\prime}\left(1,1^{\prime}\right)$. After this correction, CEID becomes equivalent to SCBA at the fourth-order. In principle, one may extend this relation between CEID and SCBA to any order in $F$. However, the amount of diagrams increases fast with increasing order so that it would not be easy to illustrate their relation in higher order case.

\subsection{Large ionic mass limit}

In the limit of infinite ionic mass, the electron density matrix is determined by equation (8) and 


$$
\dot{\mu}=\frac{1}{i \hbar}\left[h_{e}, \mu\right]-\frac{1}{i \hbar} C\left[F, \rho_{e}\right]
$$

Here the constant $C$ corresponds to the equal-time classical phonon Green's function because the oscillator with infinite mass is treated classically (see appendix). In Ref. [16], it was shown that these coupled equations of motion are identical to the corresponding kinetic equations for the following effective elastic scattering problem.

Consider non-interacting electrons linearly coupled to a single infinitely heavy classical degree of freedom $X$, with a distribution $\chi(X)=\frac{1}{2}[\delta(X-\sqrt{C})+\delta(X+\sqrt{C})]$.

The system can be described in terms of one-electron density matrix $\rho(X, t)$ which is governed by $i \hbar \dot{\rho}(X, t)=[h(X), \rho(X, t)]$ with the one-electron Hamiltonian $h(X)=h_{0}-F X$. Define

$$
\begin{gathered}
\rho_{e}(t) \equiv \int \rho(X, t) \chi(X) d X \\
\mu(t) \equiv \int X \rho(X, t) \chi(X) d X \\
\mu_{2}(t) \equiv \int X^{2} \rho(X, t) \chi(X) d X=C \rho_{e}
\end{gathered}
$$

Then $\rho_{e}(t)$ is generated exactly by

$$
\begin{gathered}
i \hbar \dot{\rho}_{e}=\left[h_{0}, \rho_{e}\right]-[F, \mu] \\
i \hbar \dot{\mu}=\left[h_{0}, \mu\right]-C\left[F, \rho_{e}\right]
\end{gathered}
$$

which are identical to equations (8) and (36) [16]. We now proceed to solve equations (37) and (38) in integral form. It is proposed that for $t>t_{0}$ this solution can be written as

$$
\rho_{e}(t)=\rho^{<}(t, t), \quad \mu(t)=\mu^{<}(t, t)
$$

where $\rho^{<}\left(t, t^{\prime}\right)$ and $\mu^{<}\left(t, t^{\prime}\right)$ are defined as

$$
\begin{gathered}
\rho^{<}\left(t, t^{\prime}\right)=-(i \hbar)^{2} \int G_{X}^{+}\left(t, t_{0}\right) \rho_{e}\left(t_{0}\right) G_{X}^{-}\left(t_{0}, t^{\prime}\right) \chi(X) d X \\
\mu^{<}\left(t, t^{\prime}\right)=-(i \hbar)^{2} \int X G_{X}^{+}\left(t, t_{0}\right) \rho_{e}\left(t_{0}\right) G_{X}^{-}\left(t_{0}, t^{\prime}\right) \chi(X) d X
\end{gathered}
$$

with $\left(i \hbar \partial_{t}-h(X)\right) G_{X}^{ \pm}\left(t, t^{\prime}\right)=\delta\left(t-t^{\prime}\right)$. Furthermore $G_{X}^{ \pm}\left(t, t^{\prime}\right)$ can be expressed in an iterative form

$$
G_{X}^{ \pm}\left(t, t^{\prime}\right)=G_{0}^{ \pm}\left(t, t^{\prime}\right)-X \int G_{0}^{ \pm}\left(t, t^{\prime \prime}\right) F\left(t^{\prime \prime}\right) G_{X}^{ \pm}\left(t^{\prime \prime}, t^{\prime}\right) d t^{\prime \prime}
$$


with $\left(i \hbar \partial_{t}-h_{0}\right) G_{0}^{ \pm}\left(t, t^{\prime}\right)=\delta\left(t-t^{\prime}\right)$ and $F(t)=\theta\left(t-t_{0}\right) F$. Repeating the use of equation (42) in equations (40) and (41), one finds that

$$
\begin{gathered}
\rho^{<}\left(t, t^{\prime}\right)=\left(1+G^{+} \cdot \Sigma_{0}^{+} \cdot\right) \rho_{0}^{<}\left(1+\cdot \Sigma_{0}^{-} \cdot G^{-}\right)+G^{+} \cdot \Sigma_{0}^{<} \cdot G^{-} \\
\mu^{<}\left(t, t^{\prime}\right)=-C \int\left(\rho_{0}^{<}\left(t, t^{\prime \prime}\right) F\left(t^{\prime \prime}\right) G^{-}\left(t^{\prime \prime}, t^{\prime}\right)+G_{0}^{+}\left(t, t^{\prime \prime}\right) F\left(t^{\prime \prime}\right) \rho^{<}\left(t^{\prime \prime}, t^{\prime}\right)\right) d t^{\prime \prime} \\
=-C \int\left(\rho^{<}\left(t, t^{\prime \prime}\right) F\left(t^{\prime \prime}\right) G_{0}^{-}\left(t^{\prime \prime}, t^{\prime}\right)+G^{+}\left(t, t^{\prime \prime}\right) F\left(t^{\prime \prime}\right) \rho_{0}^{<}\left(t^{\prime \prime}, t^{\prime}\right)\right) d t^{\prime \prime}
\end{gathered}
$$

with

$$
\begin{gathered}
\Sigma_{0}^{ \pm}\left(t, t^{\prime}\right)=C F(t) G_{0}^{ \pm}\left(t, t^{\prime}\right) F\left(t^{\prime}\right) \\
\Sigma_{0}^{<}\left(t, t^{\prime}\right)=C F(t) \rho_{0}^{<}\left(t, t^{\prime}\right) F\left(t^{\prime}\right) \\
\rho_{0}^{<}\left(t, t^{\prime}\right)=-(i \hbar)^{2} G_{0}^{+}\left(t, t_{0}\right) \rho_{e}\left(t_{0}\right) G_{0}^{-}\left(t_{0}, t^{\prime}\right) \\
G^{ \pm}\left(t, t^{\prime}\right) \equiv \int G_{X}^{ \pm}\left(t, t^{\prime}\right) \chi(X) d X=G_{0}^{ \pm}\left(t, t^{\prime}\right)+\int G_{0}^{ \pm}\left(t, t_{1}\right) \Sigma_{0}^{ \pm}\left(t_{1}, t_{2}\right) G^{ \pm}\left(t_{2}, t^{\prime}\right) d t_{1} d t_{2}
\end{gathered}
$$

Above $A=B \cdot C$ stands for $A\left(t, t^{\prime}\right)=\int B\left(t, t^{\prime \prime}\right) C\left(t^{\prime \prime}, t^{\prime}\right) d t^{\prime \prime}$. This convention will be used where appropriate hereafter.

In the infinite mass limit, the CEID equations of motion (8) and (36) have precisely the same form as the kinetic equations (37) and (38) for the elastic scattering problem. Hence, in view of the solution to the elastic scattering problem, we may, by analogy, suggest the following ansatz to equations (8) and (36) in the context of NEGF (cf. equations (44) and (45)):

$$
G_{C E I D}\left(1,1^{\prime}\right)=G_{0}\left(1,1^{\prime}\right)+C \int G_{0}(1,2)\left[F\left(r_{2}\right) G_{0}(2,3) F\left(r_{3}\right)\right] G\left(3,1^{\prime}\right) d 2 d 3
$$

and

$$
\begin{aligned}
\Gamma_{\mu}\left(1,1^{\prime}\right) & =-C \int G_{0}(1,2) F\left(r_{2}\right) G\left(2,1^{\prime}\right) d 2 \\
& =-C \int G(1,2) F\left(r_{2}\right) G_{0}\left(2,1^{\prime}\right) d 2
\end{aligned}
$$

In analogy with the treatment in section (2), one can easily verify that $\rho_{e}\left(r, t \mid r^{\prime}, t\right)=-i \hbar G_{C E I D}^{<}\left(r t, r^{\prime} t\right)$ and $\mu\left(r, t \mid r^{\prime}, t\right)=-i \hbar \Gamma_{\mu}^{<}\left(r t, r^{\prime} t\right)$ are solutions to equations (8) and (36). In the large ionic mass limit, the CEID equations of motion are thus exactly solvable based on a correspondence between CEID 
and the elastic scattering problem. Interestingly, the Dyson equation (46) is consistent with the Born approximation (BA) and $G_{C E I D}$ contains only one term at each order in $F$.

To compare with SCBA, we need the mixed quantum-classical perturbation expansion for $G_{S C B A}$ which, following the discussion in the appendix, can be obtained by replacing the quantum phonon Green's function by the classical phonon Green's function (50) in the SCBA Dyson equation $G_{S C B A}=$ $G_{0}+G_{0} \cdot F D_{0} G F \cdot G$ (the Hartree-like diagrams are ignored here). In the infinite ionic mass limit, the classical phonon Green's function (50) is a constant $C$. So the SCBA Dyson equation becomes $G_{S C B A}=G_{0}+C G_{0} \cdot F G F \cdot G$. This equation differs from the CEID solution (46) from the fourth-order term onwards. For instance, $G_{S C B A}^{(4)}=2 G_{C E I D}^{(4)}$. In the large ionic mass limit, the difference between CEID and SCBA is thus precisely quantified.

\section{Conclusions}

In this paper, we have considered, using the nonequilibrium Green's function theory, a system of noninteracting electrons linearly coupled to a quantum oscillator. A set of kinetic equations, which determine the one-electron density matrix, are derived with the equation-of-motion technique. Our work establishes a rigorous connection between CEID and NEGF, and extends the scope of CEID to a general nonequilibrium ensemble that allows for a variable total number of electrons. By perturbation theory, the decoupling approximations used in the CEID methodology can be quantified in diagrammatic terms.

We have compared the limiting behavior of CEID and SCBA analytically. In the weak electron-phonon coupling limit, they agree exactly for a general nonequilibrium state of the system. In the large ionic mass limit, where CEID corresponds to an elastic scattering problem and can be solved exactly, the difference between CEID and SCBA emerges from the fourth-order term and can be quantified. In particular, we illustrate the connection between CEID and SCBA at the fourth-order in the coupling strength. We find that, CEID occupies a special place between BA and SCBA, such that CEID is simpler than SCBA but is an improvement over BA, in that CEID conserves total number of electrons. The lowest-order SCBA corrections to the CEID equations of motion (see section 3.2) no longer involve just single-time quantities. This illustrates the sense in which CEID can be thought of as the simplest particle-number conserving approximation that, in addition, retains just single-time quantities.

The present formulation of CEID can be extended to include multiple quantum oscillators. The purpose of the single-oscillator model calculation is to illustrate the analytical features of CEID in a simple way so that an analytical comparison of CEID and SCBA can be made. Like SCBA, the present method for CEID is not applicable to problems with strong electron-phonon correlations, which, however, have been addressed by another CEID scheme [19] recently. 


\section{Acknowledgments}

The author is greatly indebted to Tchavdar Todorov for suggesting this problem, and for numerous discussions and continuous ideas. The author thanks colleagues from the EPSRC Consortium on Modelling Non-Adiabatic Processes in Materials with Correlated Electron-Ion Dynamics for sharing their insights into CEID. A critical reading of the manuscript by Lev Kantorovich and Eunan McEniry is gratefully acknowledged. This work was supported by EPSRC under Grant No. EP/C006739/01.

\section{Appendix}

In this appendix, we shall consider the Green's function for a system of quantum electrons coupled to a classical oscillator and then show how to develop a diagrammatic perturbation expansion for it. The system Hamiltonian takes the form (1) but $X$ and $P$ are classical variables now. The mixed quantum-classical Green's function, as usual, can be written as

$$
G\left(1,1^{\prime}\right)=(i \hbar)^{-1}\left\langle T_{C}\left[\Psi_{H_{0}}(1) \Psi_{H_{0}}^{+}\left(1^{\prime}\right) e^{-\frac{i}{\hbar} \int_{C} H_{H_{0}}^{i}(\tau) d \tau}\right]\right\rangle
$$

where the angular bracket $\langle\cdots\rangle=\int d X d P \operatorname{tr}\left(\rho_{0} \cdots\right)$. To evaluate this Green's function, we must provide a procedure for evaluating the average of products of classical coordinates $X$ 's, while the average of products of electronic field operators can be evaluated by Wick's theorem.

Consider a classical oscillator with position, momentum and energy

$$
\begin{gathered}
X(t)=A \cos (\omega t-\phi) \\
P(t)=-A \omega M \sin (\omega t-\phi) \\
E=\frac{P^{2}}{2 M}+\frac{1}{2} M \omega^{2} X^{2}=\frac{1}{2} M \omega^{2} A^{2}
\end{gathered}
$$

sampled from the canonical distribution

$$
\rho(X, P)=\frac{\beta \omega}{2 \pi} \mathrm{e}^{-\frac{1}{2} \beta M \omega^{2} A^{2}}
$$

Let $\langle\ldots\rangle$ denote averaging over $\rho$. Changing variables from $(X, P)$ to $(A, \phi)$ with $d X d P \rightarrow M \omega A d A d \phi$,

$$
\langle\ldots\rangle=\int_{-\infty}^{\infty} d X \int_{-\infty}^{\infty} d P \ldots \rho=\frac{\beta M \omega^{2}}{2 \pi} \int_{0}^{\infty} A d A \int_{0}^{2 \pi} d \phi \ldots \mathrm{e}^{-\frac{1}{2} \beta M \omega^{2} A^{2}}
$$

Our aim is to establish the relation 
$L \equiv\left\langle X\left(t_{1}\right) X\left(t_{2}\right) \ldots X\left(t_{2 N}\right)\right\rangle=R \equiv\left\langle X\left(t_{1}\right) X\left(t_{2}\right)\right\rangle\left\langle X\left(t_{3}\right) X\left(t_{4}\right)\right\rangle \ldots\left\langle X\left(t_{2 N-1}\right) X\left(t_{2 N}\right)\right\rangle$

$$
+ \text { all other pairings }
$$

Write

$$
X\left(t_{i}\right)=\frac{A}{2}\left(\mathrm{e}^{i\left(\omega t_{i}-\phi\right)}+\mathrm{e}^{-i\left(\omega t_{i}-\phi\right)}\right)=\frac{a_{i}+a_{i}^{*}}{2}, \quad a_{i}=A \mathrm{e}^{i\left(\omega t_{i}-\phi\right)}
$$

Consider $L$. Expand and integrate over $\phi$. Only terms with $N$ a's and $N a^{*}$ 's survive. There are

$$
N_{L}=\frac{(2 N) !}{N ! N !}
$$

such terms. Each is of the form

$$
\begin{aligned}
& \frac{1}{4^{N}}\left(\beta M \omega^{2} \int_{0}^{\infty} d A A^{2 N+1} \mathrm{e}^{-\frac{1}{2} \beta M \omega^{2} A^{2}}\right)\left(\mathrm{e}^{i \omega\left(t_{k_{1}}+\ldots+t_{k_{N}}\right)} \times \mathrm{e}^{-i \omega\left(t_{k_{N+1}}+\ldots+t_{k_{2 N}}\right)}\right) \\
& =\frac{1}{2^{N}} \frac{N !}{\left(\beta M \omega^{2}\right)^{N}}\left(\mathrm{e}^{i \omega\left(t_{k_{1}}+\ldots+t_{k_{N}}\right)} \times \mathrm{e}^{-i \omega\left(t_{k_{N+1}}+\ldots+t_{k_{2 N}}\right)}\right)
\end{aligned}
$$

with one such term occurring in $L$ for each of the $N_{L}$ possible groupings of the $2 N$ indices into two groups of $N,\left\{\left(k_{1} \ldots k_{N}\right),\left(k_{N+1} \ldots k_{2 N}\right)\right\}$.

Now consider $R$. Note that the classical phonon Green's function

$$
\left\langle X\left(t_{i}\right) X\left(t_{j}\right)\right\rangle=\frac{1}{\beta M \omega^{2}} \cos \omega\left(t_{i}-t_{j}\right)=\frac{1}{2 \beta M \omega^{2}}\left(\mathrm{e}^{i \omega\left(t_{i}-t_{j}\right)}+\mathrm{e}^{-i \omega\left(t_{i}-t_{j}\right)}\right)
$$

In $R$ there are

$$
N_{P}=(2 N-1)(2 N-3) \ldots 1=\frac{(2 N) !}{2^{N} N !}
$$

different pairings. Each pairing contributes $2^{N}$ terms, each of the form

$$
\frac{1}{2^{N}} \frac{1}{\left(\beta M \omega^{2}\right)^{N}}\left(\mathrm{e}^{i \omega\left(t_{k_{1}}+\ldots+t_{k_{N}}\right)} \times \mathrm{e}^{-i \omega\left(t_{k_{N+1}}+\ldots+t_{k_{2 N}}\right)}\right)
$$

Thus, $R$ is composed of

$$
N_{R}=\frac{(2 N) !}{2^{N} N !} 2^{N}=\frac{(2 N) !}{N !}
$$

terms, each of the form (51).

By symmetry, every grouping of indices $\left\{\left(k_{1} \ldots k_{N}\right),\left(k_{N+1} \ldots k_{2 N}\right)\right\}$ that occurs in $L$ occurs in $R$ and vice versa. Further, by symmetry, if a given grouping $\left\{\left(k_{1} \ldots k_{N}\right),\left(k_{N+1} \ldots k_{2 N}\right)\right\}$ occurs $G$ times in $R$, then every other grouping must also occur $G$ times in $R$. There are $N_{L}$ groupings. Hence 


$$
G=\frac{N_{R}}{N_{L}}=N !
$$

and thus every grouping $\left\{\left(k_{1} \ldots k_{N}\right),\left(k_{N+1} \ldots k_{2 N}\right)\right\}$ occurs $N$ ! times in $R$. Using this and (51), we see that in $R$ each distinct grouping occurs with a prefactor

$$
\frac{1}{2^{N}} \frac{N !}{\left(\beta M \omega^{2}\right)^{N}}
$$

which is the same as the prefactor with which each grouping occurs in $L$ (see equation (49)). Hence $L=R$. This relation (Wick's theorem) allows us to evaluate the mixed quantum-classical Green's function as a perturbation expansion involving only wholly paired nuclear coordinates. 


\section{References}

[1] For a review, see, N. Agraït, A. L. Yeyati, and J. M. van Ruitenbeek, Phys. Rep. 377, 81 (2003); also, M. Galperin, M. A. Ratner, and A. Nitzan, J. Phys.: Condens. Matter 19, 103201 (2007).

[2] A. P. Horsfield, D. R. Bowler, A. J. Fisher, T. N. Todorov, and C. G. Sánchez, J. Phys.: Condens. Matter 17, 4793 (2005).

[3] T. N. Todorov, Phil. Mag. B 77, 965 (1998).

[4] L. V. Keldysh, Sov. Phys. JETP 20, 1018 (1965); L. V. Keldysh, in Progress in Nonequilibrium Green's Functions, edited by M. Bonitz and D. Semkat (World Scientific, Singapore, 2003).

[5] H. Haug and A. P. Jauho, Quantum Kinetics in transport and Optics of Semiconductors (Springer, Berlin, 1996).

[6] J. Rammer, Quantum Field Theory of Non-Equilibrium States (Cambridge University Press, New York, 2007).

[7] C. Caroli, R. Combescot, P. Nozieres, and D. Saint-James, J. Phys. C: Solid State Phys. 5, 21 (1972).

[8] P. Hyldgaard, S. Hershfield, J. H. Davies, and J. W. Wilkins, Ann. Phys. (N.Y.) 236, 1 (1994).

[9] M. Galperin, M. A. Ratner, and A. Nitzan, J. Chem. Phys. 121, 11965 (2004).

[10] T. Frederiksen, M. Paulsson, M. Brandbyge, and A. P. Jauho, Phys. Rev. B 75, 205413 (2007).

[11] G. L. Goodvin, M. Berciu, and G. A. Sawatzky, Phys. Rev. B 74, 245104 (2006).

[12] A. P. Horsfield, D. R. Bowler, A. J. Fisher, T. N. Todorov, and C. G. Sánchez, J. Phys.: Condens. Matter 16, 8251 (2004).

[13] A. P. Horsfield, D. R. Bowler, H. Ness, C. G. Sánchez, T. N. Todorov, and A. J. Fisher, Rep. Prog. Phys. 69, 1195 (2006).

[14] E. J. McEniry, D. R. Bowler, D. Dundas, A. P. Horsfield, C. G. Sánchez, and T. N. Todorov, J. Phys.: Condens. Matter 19, 196201 (2007).

[15] D. Dundas, E. J. McEniry, and T. N. Todorov, Nature Nanotech. 4, 99 (2009).

[16] E. J. McEniry, T. Frederiksen, T. N. Todorov, D. Dundas, and A. P. Horsfield, Phys. Rev. B 78, 035446 (2008). 
[17] T. N. Todorov, G. A. D. Briggs, and A. P. Sutton, J. Phys.: Condens. Matter 5, 2389 (1993).

[18] T. N. Todorov, J. Phys.: Condens. Matter 14, 3049 (2002).

[19] L. Stella, M. Meister, A. J. Fisher, and A. P. Horsfield, J. Chem. Phys. 127, 214104 (2007). 


\section{Figure captions}

Figure 1. (a) Zero- and second-order noncrossing diagrams in the exact $\Gamma_{\mu_{2}}^{\prime}\left(r t, r^{\prime} t^{\prime}\right)$ (see equation (14)). (b) The diagrammatic representation of the CEID decoupling approximation for $\Gamma_{\mu_{2}}^{\prime}\left(r t, r^{\prime} t^{\prime}\right)$ (see equation (16)). The thick (thin) straight line represents dressed (bare) electron Green's function. The thick (thin) wavy line represents dressed (bare) phonon Green's function. We ignore Hartree-like diagrams and corrections to a phonon line.

Figure 2. The diagrammatic representation of the fourth-order SCBA Green's function $G^{(4)}\left(1,1^{\prime}\right)$. 
(a)

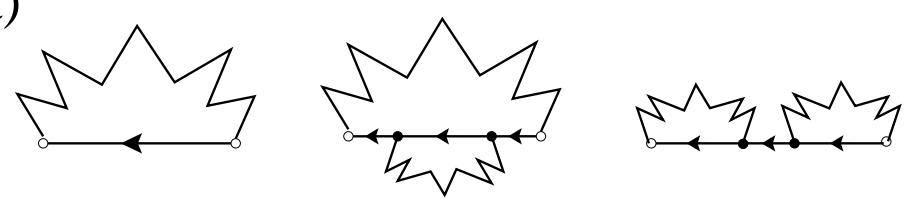

(b)

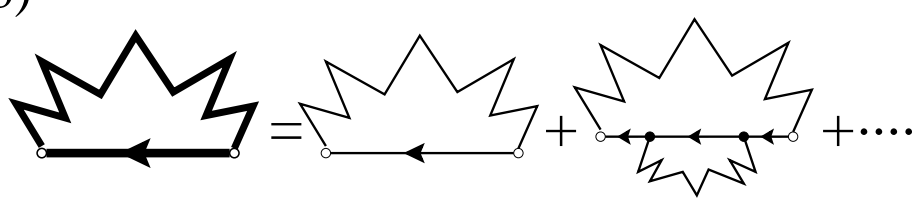

Y. Wang, Figure 1 
(a)

(b)
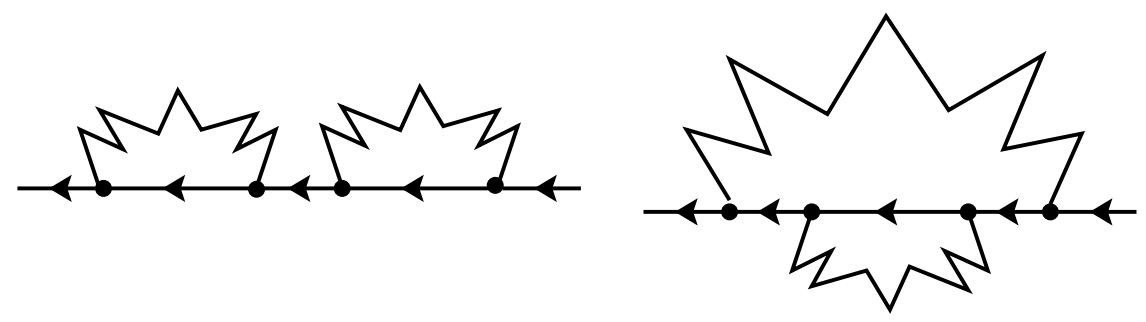

Y. Wang, Figure 2 